\title{
A COUNTEREXAMPLE TO AN F. AND M. RIESZ-TYPE THEOREM KRZYSZTOF SAMOTIJ
}

(Communicated by Irwin Kra)

\begin{abstract}
A premeasure is a finitely additive complex-valued function $\mu$ defined on the semiring of all connected subsets of $\mathbf{T}$, continuous at $\varnothing$ and with $\mu(\varnothing)=\mu(\mathbf{T})=0$. Let $\kappa$ be a continuous increasing concave function on $[0,2 \pi]$ with $\kappa(0)=0$. A conjecture from [3] saying that if the Poisson integral of a premeasure $\mu$ is holomorphic in the open unit disk and $\operatorname{Var}_{\kappa}(\mu)<\infty$ then $\lim _{\tau \rightarrow 0} \operatorname{Var}_{\kappa}\left(\mu_{\tau}-\mu\right)=0$ is disproved, where $\operatorname{Var}_{\kappa}(\mu)=$ $\sup \sum_{j}\left|\mu\left(I_{j}\right)\right| / \sum_{j} \kappa\left(\left|I_{j}\right|\right)$ (the supremum is taken over all finite partitions of $\mathbf{T}$ into connected subsets $I_{j}$ ) and $\mu_{\tau}$ denotes the $\tau$-translation of $\mu$.
\end{abstract}

In this paper a counterexample to a conjecture of $\mathrm{B}$. Korenblum is given. Following [2] and [3] we introduce the following notations.

$\mathbf{D}$ denotes the open unit disk in the complex plane and $\mathbf{T}$ is the unit circle. I denotes the family of all intervals in $\mathbf{T}$ (i.e. of all connected subsets of $\mathbf{T}$ ). A function $\mu: I \rightarrow \mathbf{C}$ is called a premeasure if it satisfies the following conditions:

(i) $\mu(\varnothing)=\mu(\mathbf{T})=0$,

(ii) $\mu\left(I_{1} \cup I_{2}\right)=\mu\left(I_{1}\right)+\mu\left(I_{2}\right)$ for all $I_{1}, I_{2} \in I$ such that $I_{1} \cap I_{2}=\varnothing$ and $I_{1} \cup I_{2} \in I$,

(iii) if $I_{1} \supseteq I_{2} \supseteq \cdots$ is a nonincreasing sequence of intervals and $\bigcap I_{n}=\varnothing$, then $\lim _{n} \mu\left(I_{n}\right)=0$.

$\kappa$ will always denote an increasing concave continuous function on $[0,2 \pi]$ such that $\kappa(0)=0$ and $\kappa(2 \pi)=1$. The $\kappa$-variation of a premeasure $\mu$ is

$$
\operatorname{Var}_{\kappa} \mu=\sup \frac{\sum_{1}^{n}\left|\mu\left(I_{j}\right)\right|}{\sum_{1}^{n} \kappa\left(\left|I_{j}\right|\right)}
$$

here $\left|I_{j}\right|$ denotes the length of the interval $I_{j}$, and the supremum is taken over all finite systems $\left\{I_{j}\right\}$ of mutually disjoint intervals $I_{j}$ such that $\bigcup I_{j}=\mathbf{T}$. If $\operatorname{Var}_{\kappa} \mu<\infty, \mu$ will be called a premeasure of bounded $\kappa$-variation. Let $V_{\kappa}$ denote the family of all premeasures of bounded $\kappa$-variation. $V_{\kappa}$ with the norm $\operatorname{Var}_{\kappa}$ is a Banach space. If $\mu$ is a premeasure and $\tau$ is a real number then the $\tau$-translation of $\mu$ is defined by

$$
\mu_{\tau}(I)=\mu\left(\left\{\xi \in \mathbf{T}: e^{i \tau} \xi \in I\right\}\right), \quad I \in I .
$$

A premeasure $\mu \in V_{\kappa}$ is called $\kappa$-absolutely continuous if $\lim _{\tau \rightarrow 0} \operatorname{Var}_{\kappa}\left(\mu-\mu_{\tau}\right)=0$.

Received by the editors January 30, 1986 and, in revised form, October 27, 1986.

1980 Mathematics Subject Classification (1985 Revision). Primary 30H05.

Key words and phrases. Poisson integral, analytic measures. 
If $f$ is a $C^{1}$ function on $\mathbf{T}$ and $\mu$ is a premeasure then we can define the integral of $f$ with respect to $\mu$ by the formula

$$
\int_{T} f(\xi) d \mu(\xi)=\int_{0}^{2 \pi} f\left(e^{i t}\right) d \tilde{\mu}(t)
$$

here $\tilde{\mu}(t)=\mu((0, t])$. Therefore we can define the Poisson integral of $\mu$ by the formula

$$
u(z)=u_{\mu}(z)=\int_{\mathbf{T}} \frac{1-|z|^{2}}{|\xi-z|^{2}} d \mu(\xi), \quad z \in \mathbf{D} .
$$

On the other hand, if $v$ is a harmonic function on $\mathbf{D}$ with $v(0)=0$ then a family $\left\{\mu^{(r)}\right\}_{r \in(0,1)}$ of premeasures is associated with $v$ by the formula

$$
\mu^{(r)}(I)=\frac{1}{2 \pi} \int_{J_{I}} v\left(r e^{i t}\right) d t, \quad I \in I
$$

here $J_{I}=\left\{t \in[0,2 \pi]: e^{i t} \in I\right\}$. It is not hard to see that if $u$ is the Poisson integral of a $\kappa$-absolutely continuous premeasure $\mu$, then

$$
\operatorname{Var}_{\kappa} \mu^{(r)} \leq \operatorname{Var}_{\kappa} \mu, \quad r \in(0,1) .
$$

The following lemma was essentially proved in [1, §5].

LEMMA. Let $u$ be a harmonic function in $\mathbf{D}$ with $u(0)=0$. Suppose that there is a constant $C$ such that $\left|\mu^{(r)}(I)\right| \leq C \kappa(|I|)$ for each $r \in(0,1)$ and each $I \in I$, where $\left\{\mu^{(r)}\right\}_{r \in(0,1)}$ is the family of premeasures associated with $u$. Then $u$ is the Poisson integral of some premeasure of $\kappa$-variation less than or equal to $C$.

A premeasure $\mu$ is said to be analytic if its Poisson integral is holomorphic in $D$ or, equivalently, if $\int \xi^{n} d \mu(\xi)=0, n=1,2, \ldots$ It was conjectured in [3] that each analytic premeasure of bounded $\kappa$-variation is always $\kappa$-absolutely continuous. The following theorem shows that this is not true except for the classical case of the theorem of F. and M. Riesz (i.e. when $\lim _{s \rightarrow 0+} \kappa(s) / s<+\infty$ ).

THEOREM. Let $\kappa$ be an increasing concave continuous function on $[0,2 \pi]$ such that $\kappa(0)=0, \kappa(2 \pi)=1$ and $\lim _{s \rightarrow 0+} \kappa(s) / s=+\infty$. Then there exists an analytic premeasure of $\kappa$-bounded variation which is not $\kappa$-absolutely continuous.

PROOF. Let $\kappa$ be as in the assumption. It is not hard to see that there exist increasing sequences $\left\{N_{n}\right\}$ of positive integers, and $\left\{r_{n}\right\}$ of positive real numbers tending to 1 , such that if we denote $\alpha_{n}=\kappa\left(\pi / N_{n}\right) / 3$, then

(i) $\alpha_{n+1} \leq \alpha_{n} / 2, n=1,2, \ldots$,

(ii) $\lim _{n \rightarrow \infty} \sum_{k=n+1}^{\infty} \alpha_{k} N_{k} r_{n}^{N_{k}}=0$,

(iii) $\lim _{n \rightarrow \infty} \max _{t}\left[\sum_{k=1}^{n-1}\left|\alpha_{k} N_{k}\left(e^{i N_{k} t}-e^{i N_{k}\left(t+\pi / N_{n}\right)}\right)\right|\right]=0$,

(iv) $\sum_{k=1}^{n-1} N_{k} \alpha_{k} \leq \alpha_{n} N_{n}, n=1,2, \ldots$,

(v) $r_{n}^{N_{n}} \geq \frac{1}{2}, n=1,2, \ldots$.

Let $f_{n}(t)=\min \left\{\alpha_{n},\left(\alpha_{n} N_{n} / \pi\right) t\right\}$, and let $\tilde{\kappa}(t)=\sum_{n=1}^{\infty} f_{n}(t), t \in[0,2 \pi]$. From (i) we deduce that $\tilde{\kappa}$ is a well-defined continuous concave function. It is constant on $\left[\pi / N_{1}, 2 \pi\right]$ and linear on each of the intervals $\left[\pi / N_{n+1}, \pi / N_{n}\right], n=1,2, \ldots$. Moreover, for each positive integer $n$ we have

$$
\tilde{\kappa}\left(\frac{\pi}{N_{n}}\right)=\sum_{k=1}^{n} \frac{\alpha_{k} N_{k}}{N_{n}}+\alpha_{n}+\sum_{k=n+1}^{\infty} \alpha_{k} .
$$


By (iv) the first term of this sum does not exceed $\alpha_{n}$. Neither, by (i), does the third. Therefore $\tilde{\kappa}\left(\pi / N_{n}\right) \leq 3 \alpha_{n}=\kappa\left(\pi / N_{n}\right)$. Since $\kappa$ is increasing and concave we have

$$
\tilde{\kappa}(t) \leq \kappa(t), \quad 0 \leq t \leq 2 \pi .
$$

Let $u(z)=\sum_{n=1}^{\infty} \alpha_{n} N_{n} z^{N_{n}}, z \in \mathbf{D}$. Since $\lim _{n \rightarrow \infty} r_{n}=1$ and by (ii), the function $u$ is well defined and holomorphic in D. Let $\left\{\mu^{(r)}\right\}_{r \in(0,1)}$ be the family of premeasures associated with $u$, as defined in (1). For arbitrary $r \in(0,1)$, arbitrary $I \in I$, and each $n$ with $r_{n} \geq r$ we have

$$
\begin{aligned}
\left|\mu^{(r)}(I)\right| \leq & \sum_{k=1}^{n}\left|\frac{1}{2 \pi} \int_{J_{I}} \alpha_{k} N_{k} r^{N_{k}} e^{i N_{k} t} d t\right| \\
& +|I| \sum_{k=n+1}^{\infty} \alpha_{k} N_{k} r_{n}^{N_{k}}
\end{aligned}
$$

But

$$
\left|\frac{1}{2 \pi} \int_{J_{I}} \alpha_{k} N_{k} r_{n}^{N_{k}} e^{i N_{k} t} d t\right| \leq \min \left\{\alpha_{k}, \frac{\alpha_{k} N_{k}}{\pi}|I|\right\} .
$$

Hence, by the definition of $f_{k}$ 's and by (ii), $\left|\mu^{(r)}(I)\right| \leq \sum_{k=1}^{n} f_{k}(|I|)+|I| c_{n}$, where $\lim _{n \rightarrow \infty} c_{n}=0$.

By (3), letting $n$ tend to infinity in the above we obtain $\left|\mu^{(r)}(I)\right| \leq \kappa(|I|)$. Thus the lemma implies that the function $u$ is the Poisson integral of some (obviously analytic) premeasure $\mu$ with $\operatorname{Var}_{\kappa} \mu \leq 1$.

For an arbitrary fixed $n$ let

$$
u_{1}(z)=\sum_{k=1}^{n-1} \alpha_{k} N_{k} z^{N_{k}}, \quad u_{2}(z)=\alpha_{n} N_{n} z^{N_{n}}
$$

and

$$
u_{3}(z)=\sum_{k=n+1}^{\infty} \alpha_{k} N_{k} Z^{N_{k}}, \quad z \in \mathbf{D} .
$$

Let $\left\{\nu^{(r)}\right\},\left\{\eta^{(r)}\right\},\left\{\chi^{(r)}\right\}$ denote the associated families of premeasures corresponding to $u_{1}, u_{2}, u_{3}$ respectively. Denote $\nu^{(n)}=\nu^{\left(r_{n}\right)}, \eta^{(n)}=\eta^{\left(r_{n}\right)}$ and $\chi^{(n)}=\chi^{\left(r_{n}\right)}$. Note that

$$
\begin{aligned}
\operatorname{Var}_{\kappa}\left(\mu^{\left(r_{n}\right)}-\mu_{\pi / N_{n}}^{\left(r_{n}\right)}\right) \geq & \operatorname{Var}_{\kappa}\left(\eta^{(n)}-\eta_{\pi / N_{n}}^{(n)}\right) \\
& -\operatorname{Var}_{\kappa}\left(\nu^{(n)}-\nu_{\pi / N_{n}}^{(n)}\right)-2 \operatorname{Var}_{\kappa} \chi^{(n)} .
\end{aligned}
$$

Observe that for every harmonic function $v$ on $\mathbf{D}$ with $v(0)=0$ we have the inequality $\operatorname{Var}_{\kappa} \lambda^{(r)} \leq \sup \{|v(z)|:|z|=r\}$, where $\left\{\lambda^{(r)}\right\}_{r \in(0,1)}$ is the family of premeasures associated with $v$. If we apply this observation to $u_{3}$ then, by (ii), we have

$$
\lim _{n \rightarrow \infty} \operatorname{Var}_{\kappa} \chi^{(n)}=0
$$

The same observation applied to $v(z)=u_{1}(z)-u_{1}\left(z e^{i \pi / N_{n}}\right)$ together with (iii) yield

$$
\lim _{n \rightarrow \infty}\left(\nu^{(n)}-\nu_{\pi / N_{n}}^{(n)}\right)=0 .
$$


Now, if we take $I_{j}=\left\{e^{i t}:(j-1) \pi / N_{n} \leq t \leq j \pi / N_{n}\right\}, j=1,2, \ldots, 2 N_{n}$, in the definition of $\kappa$-variation then we obtain

$$
\begin{aligned}
\operatorname{Var}_{\kappa}\left(\eta^{(n)}\right. & \left.-\eta_{\pi / N_{n}}^{(n)}\right) \geq \frac{\sum_{j=1}^{2 N_{n}}\left|\eta^{(n)}\left(I_{j}\right)-\eta_{\pi / N_{n}}^{(n)}\left(I_{j}\right)\right|}{\sum_{j=1}^{2 N_{n}} \kappa\left(\left|I_{j}\right|\right)} \\
& =\frac{1}{2 N_{n} \kappa\left(\pi / N_{n}\right)} \sum_{j=1}^{2 N_{n}} \frac{\alpha_{n} N_{n} r_{n}^{N_{n}}}{2 \pi}\left|\int_{(j-1) \pi / N_{n}}^{j \pi / N_{n}}\left(e^{i N_{n} t}-e^{i N_{n}\left(t+\pi / N_{n}\right)}\right) d t\right| \\
& \geq \frac{1}{6}
\end{aligned}
$$

where the last inequality follows by (v). This together with (6), (5), and (4) imply

$$
\varliminf_{n \rightarrow \infty} \operatorname{Var}_{\kappa}\left(\mu^{\left(r_{n}\right)}-\mu_{\pi / N_{n}}^{\left(r_{n}\right)}\right)>0 .
$$

If $\mu$ was $\kappa$-absolutely continuous then (7) and (2) would imply that

$$
\varliminf_{n \rightarrow \infty} \operatorname{Var}_{\kappa}\left(\mu-\mu_{\pi / N_{n}}\right)>0
$$

which contradicts the $\kappa$-absolute continuity of $\mu$.

\section{REFERENCES}

1. B. Korenblum, An extension of Nevanlinna theory, Acta Math. 135 (1975), 187-219.

2. _ A generalization of two classical convergence tests for Fourier series, and some new Banach spaces of functions, Bull. Amer. Math. Soc. (N.S.) 9 (1983), 215-218.

3. , On a class of Banach spaces associated with the notion of entropy, Trans. Amer. Math. Soc. 290 (1985), 527-553.

INSTytut MATEMATYKI, POLITEChNiKa WroclaWSKa, WybRzeże WyspianSKIEgO 27, 50-370 WROCLAW, POLAND 